\title{
Mortalidad materna en Guatemala: diferencias
entre muerte hospitalaria y no hospitalaria
}

\author{
Ana Marina Tzul, MD MSP,(') Edgar Kestler, PhD,(2) Bernardo Hernández-Prado, D en C, (3) \\ Carlos Hernández-Girón, D en C. ${ }^{(3)}$
}

Tzul AM, Kestler E, Hernández-Prado B, Hernández-Girón C. Mortalidad materna en Guatemala: diferencias entre muerte hospitalaria y no hospitalaria. Salud Publica Mex 2006;48:183-192.

\section{Resumen}

Objetivo. Estimar la asociación entre características obstétricas, sociodemográficas y factores de riesgo relacionados con la mortalidad materna hospitalaria y no hospitalaria en Guatemala durante el año 2000. Material y métodos. Se realizó un estudio epidemiológico transversal con 649 casos de muertes maternas (MM) ocurridas en la República de Guatemala durante el año 2000, en el que se compararon las características de las muertes maternas hospitalarias y no hospitalarias. Resultados. De 649 MM registradas, 270 (4I.6\%) se clasificaron como MM hospitalarias y 379 (58.4\%) como MM no hospitalarias. La mayor proporción de muertes ocurrió en mujeres mayores de 35 años de edad $(29.28 \%)$, indígenas $(65.49 \%)$, casadas o unidas $(87.83 \%)$, con ocupación no remunerada (94.78\%), sin educación $(66.56 \%)$. El riesgo de $M M$ no hospitalaria fue mayor en mujeres del grupo indígena $(R M=3.4 ;$ IC95\% 2.8-5.3), con ocupación no remunerada $(R M=8.95 ;$; IC95\% I.7-46.4), bajo nivel escolar $(R M=1.96 ;$ IC95\% I.0-3.8), y hemorragia como causa básica de muerte $(R M=4.28$; IC95\% 2.3-7.9). Conclusiones. De los 679 casos de MM ocurridas en Guatemala en el año $2000,58 \%$ correspondió a MM no hospitalarias, lo que puede estar relacionado con el hecho de que una alta proporción de la población habita en áreas rurales o de alta marginalidad, además de aspectos culturales (mayoría indígena) que dificultan la accesibilidad a los servicios de salud. Los resultados presentados pueden servir de orientación para determinar estrategias de intervención que prevengan la mortalidad materna en los ámbitos hospitalario y extrahospitalario, en Guatemala.

Palabras clave: muerte materna; factores de riesgo; epidemiología; Guatemala
Tzul AM, Kestler E, Hernández-Prado B, Hernández-Girón C. Maternal mortality in Guatemala: differences between hospital and non-hospital deaths.

Salud Publica Mex 2006;48:183-192.

\begin{abstract}
Objective: To estimate the association between obstetric and socio-demographic characteristics and risk factors related to intra- and extra-hospital maternal mortality in Guatemala during the year 2000. Materials and Methods: A cross-sectional epidemiologic study was carried out in 649 maternal mortality (MM) cases that occurred in Guatemala during 2000, comparing characteristics of intra- and extrahospital maternal deaths. Multivariate statistical analysis was conducted using Stata 7.0 software. Results: Out of 649 registered $M M$ cases, 270 (41.6\%) were classified as intrahospital MM and 379 (58.4\%) as extra-hospital MM.A larger proportion of deaths occurred in women over 35 years of age $(29.28 \%)$, those of indigenous ethnicity $(65.49 \%)$, married or cohabiting (87.83\%), who had unpaid employment (94.78\%), and without formal education (66.56\%). Compared with intra-hospital MM cases, the risk of extra-hospital MM was greater among indigenous women (OR 3.4; CI95\% 2.8-5.3), those who had unpaid employment (OR 8.95; Cl95\% I.7-46.4), a low level of formal education (OR I.96; C195\% I.0-3.8) and hemorrhaging as the immediate cause of death (OR 4.28; CI95\% 2.3-7.9).Conclusions: Although some characteristics of intra- and extra-hospital MM cases are similar, a greater proportion of deaths were extra-hospital. This could be related to the high percentage of the population that lives in rural or marginalized areas, which in addition to certain cultural aspects (related to the fact that most of the population is indigenous) may impede access to health services. The results of this study can be useful for determining intervention strategies to prevent maternal mortality in intra- and extra-hospital contexts in Guatemala.
\end{abstract}

Key words: maternal mortality; risk factors; epidemiology; Guatemala

(I) Escuela de Salud Pública de México. Instituto Nacional de Salud Pública, México.

(2) Centro de Investigación Epidemiológica en Salud Sexual y Reproductiva. Ministerio de Salud Pública y Asistencia Social, Guatemala.

(3) Dirección de Salud Reproductiva, Centro de Investigación Salud Poblacional. Instituto Nacional de Salud Pública, México.

Fecha de recibido: 25 de julio de 2005 - Fecha de aceptado: 10 de marzo de 2006

Solicitud de sobretiros: Dr. Carlos Hernández-Girón. Centro de Investigación en Salud Poblacional, Avenida Universidad 655, Colonia Santa María Ahuacatitlán, 62508. Cuernavaca, Morelos, México. Correo electrónico: chernand@correo.insp.mx 
E panorama epidemiológico mundial de la mortalidad materna muestra contrastes importantes. A pesar de la tendencia descendente, las diferencias entre regiones y países son importantes. En países en vías de desarrollo, la razón de mortalidad materna (RMM) es 480 muertes maternas (MM) por 100000 nacidos vivos (NV). En el ámbito mundial, América presenta una de las mayores desigualdades de mortalidad materna, lo cual se refleja al comparar la RMM de Canadá, con cuatro casos de MM por $100000 \mathrm{NV}$, con la de Haití, con 523 casos de MM por 100000 NV. Guatemala se encuentra entre los 11 países con los más altos índices de mortalidad materna de Latinoamérica. ${ }^{1-4}$

Según la Organización Mundial de la Salud (OMS) una mujer embarazada en un país en vías de desarrollo tiene 100 a 200 veces más riesgo de morir que una mujer en un país desarrollado.,

Diferentes estudios destacan la influencia que los factores sociodemográficos y obstétricos ejercen sobre la ocurrencia de la muerte materna, como son la edad, etnia, estado civil, escolaridad, número de gestaciones, periodo del embarazo, lugar del parto y persona que lo asiste, accesibilidad a servicios de salud y utilización de los mismos. En Guatemala, para el año 2000, los datos informan que $73.5 \%$ de los partos ( $\mathrm{N}=425410)$ ocurrió en la casa de la parturienta y sólo $26.35 \%$ tuvo lugar en los hospitales. Estas características determinan importantes diferencias entre MM hospitalaria y no hospitalaria. ${ }^{1,2,6-8}$

A partir de los datos generados en la encuesta Línea Basal de Mortalidad Materna (LBMM 2000) se pretende identificar los factores relacionados con la ocurrencia de 649 casos de MM registradas durante el año 2000 en Guatemala, analizando las diferencias entre MM ocurridas en los servicios médicos (hospitalaria) y las ocurridas en el domicilio (no hospitalarias).

\section{Material y métodos}

Para la elaboración de la Línea Basal de Mortalidad Materna (LBMM), realizada por el Ministerio de Salud de Guatemala, se tomó en cuenta el total de mujeres que fallecieron en ese país durante el año 2000 (4 416 muertes). De esta base, se identificó a un total de 649 muertes maternas (MM) a partir de los certificados de defunción (de los registros municipales) de mujeres en edad fértil (10 a 49 años de edad). Para estos casos que se notificaron como MM se revisaron los datos de los certificados de defunción y del historial clínico de pacientes que fallecieron en los hospitales; por otra parte, se realizó autopsia verbal en los casos de MM que ocurrieron tanto en el hospital como en el domicilio $\mathrm{u}$ otro sitio.
Se incluyó en el estudio a todas las muertes de mujeres entre 14 y 49 años de edad que fallecieron por causas maternas en la República de Guatemala durante el periodo del 1 de enero al 31 de diciembre del año 2000. Se excluyeron los casos de mujeres en edad fértil muertas por causas diferentes a la materna y mujeres embarazadas fallecidas por causas diferentes a la materna.

Para la recolección de los datos se utilizaron tres instrumentos diferentes:

1. Registro de muerte: para identificar todas las muertes de mujeres en edad fértil en los registros civiles de todos los municipios y departamentos de Guatemala, se procedió a revisar los datos de toda mujer con causa de muerte relacionada con el embarazo, parto o puerperio y que estuviera en edad fértil (14-49 años). La información se recolectó por medio de una boleta (Formulario A) que comprendía datos generales e historia obstétrica. Luego se procedió a llenar dos formularios adicionales.

2. Autopsia verbal de la muerte: aplicado por un médico del Ministerio de Salud, durante el primer mes después de ocurrida la muerte, en la casa de habitación de las mujeres, al familiar más cercano, comprendía: datos generales de la paciente y características ginecobstétricas y de atención del parto.

Para proceder a realizar la autopsia verbal se solicitó el consentimiento informado de la persona que fungió como informante (familiar más cercano).

3. Formulario de codificación de datos de la muerte materna: se tomó como referencia de muerte materna la definición que se encuentra en la $10^{a}$ edición de la Clasificación Internacional de Enfermedades ("muerte materna es la muerte de una mujer mientras está embarazada o dentro de los 42 días siguientes a la terminación del embarazo, independientemente de la duración y el sitio del embarazo, debida a cualquier causa relacionada con o agravada por el embarazo mismo o su atención, pero no por causas accidentales o incidentales" ${ }^{\prime \prime}{ }^{9}$ Se codificaron los datos obtenidos y de esa manera se obtuvo el diagnóstico preciso de la causa de muerte.

De acuerdo con el lugar de ocurrencia de la muerte se formaron dos grupos de comparación, los cuales se definieron de la siguiente manera:

- Muertes maternas hospitalarias $(n=270)$ : aquellas muertes que en la base de datos registren el lugar de ocurrencia como hospitales públicos o privados (casos que tuvieron registro de ingreso hospitalario en centro de atención de segundo y tercer nivel). 
- Muertes maternas no hospitalarias $(n=379)$ : aquellas muertes en las que se registró en la base de datos el lugar de ocurrencia como las casas donde vivían las difuntas, vía pública y otros (casos que no tuvieron registro de ingreso hospitalario).

Como variable dependiente se analizó el sitio de ocurrencia de la muerte materna (hospitalaria y no hospitalaria) y como variables independientes se analizaron factores socioeconómicos (edad, estado civil, grupo étnico, ocupación, grado de educación, lugar de residencia, persona que asistió el parto) y factores clínicos (número de embarazos, edad gestacional, resultado del embarazo, tipo de parto, causa de muerte, condición asociada a la muerte, días entre la muerte y la finalización del embarazo y la clasificación final de la muerte).

Para el análisis estadístico se realizó un análisis descriptivo, se utilizaron pruebas $t$ y ji cuadrada para variables continuas y categóricas, respectivamente. Para estimar el riesgo de muerte no hospitalaria asociado a las variables sociodemográficas y obstétricas de interés se calcularon razones de momios $(R M)$ cruda y ajustada con un intervalo de confianza (IC) de $95 \%$, por medio de un análisis bivariado y multivariado, y se ajustaron modelos de regresión logística que permitieran relacionar las variables con la ocurrencia de muertes materna no hospitalarias. Para el análisis se utilizó el programa estadístico STATA 7.*

* Stata Corporation. Versión 7.0 Stata . College Station, 2002.

\section{Resultados}

En Guatemala, en el año 2000, se registraron 649 muertes maternas (MM), que corresponden a una razón de mortalidad materna de 153 por 100000 nacidos vivos. Para este estudio los casos de MM se clasificaron en dos grupos: 270 (41.6\%) muertes hospitalarias y 379 $(58.4 \%)$ muertes no hospitalarias.

\section{Características sociodemográficas}

La mayor proporción de muertes ocurrió en mujeres mayores de 35 años de edad ( $30.87 \%$ no hospitalarias y $27.0 \%$ hospitalarias). Referente a la media de edad de las mujeres fallecidas, clasificadas como MM hospitalaria (28.4 años) y MM no hospitalaria (29.2 años), no se encontró diferencia de significación estadística $(p=>0.05)$ (cuadros I y II).

La mayor proporción de muertes, tanto en hospitales $(41.48 \%)$ como fuera de éstos $(82.59 \%)$, sucedió en mujeres indígenas; en relación con el estado civil, en ambos grupos la mayor proporción de mujeres estaba casada o unida (hospitalario $79.77 \%$ y no hospitalario $92.77 \%$ ). La proporción de mujeres con una ocupación no remunerada fue alta en ambos grupos, $98.9 \%$ en MM no hospitalarias y $88.8 \%$ hospitalarias $(88.76 \%)$ (cuadro II).

El grado de educación fue bajo en ambos grupos. La proporción de analfabetismo fue $63 \%$ en las MM hospitalarias y $69 \%$ en las MM no hospitalarias. La media de años de escolaridad fue 2.1 y 1.1 años, respectivamente. Esta diferencia fue estadísticamente significativa $(p<0.05)$ (cuadros I y II).

\section{Cuadro I}

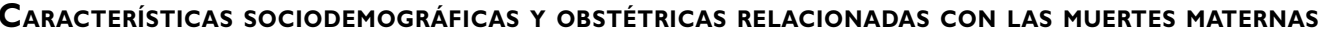
HOSPITALARIAS Y NO HOSPITALARIAS. GUATEMALA, 2000

\begin{tabular}{|c|c|c|c|c|c|c|c|c|c|}
\hline \multirow[b]{2}{*}{ Factores relacionados } & \multicolumn{4}{|c|}{ Muerte hospitalaria $(n=270)$} & \multicolumn{4}{|c|}{ Muerte no hospitalaria $(n=379)$} & \multirow[b]{2}{*}{ Valor $p$} \\
\hline & $n$ & Media & $D E^{*}$ & Rango & $n$ & Media & $D E^{*}$ & Rango & \\
\hline Edad (años) & 270 & 28.42 & 7.87 & $16-46$ & 379 & 29.23 & 8.14 & $14-48$ & 0.2054 \\
\hline Educación (años) & 270 & 2.11 & 3.51 & $0-15$ & 379 & 1.12 & 2.25 & $0-15$ & 0.0001 \\
\hline Gestaciones previas (embarazos) & 241 & 3.51 & 3.49 & $0-18$ & 371 & 4.66 & 3.63 & $0-20$ & 0.0001 \\
\hline Edad gestacional (semanas) & 185 & 33.82 & 9.81 & $4-42$ & 300 & 37.72 & 6.29 & $8-42$ & 0.0000 \\
\hline Tiempo entre resolución del embarazo y muerte (días) & 213 & 5.59 & 22.18 & $0-311$ & 328 & 1.93 & 6.14 & $0-54$ & 0.0194 \\
\hline Tiempo entre ingreso hospitalario y muerte (días) $^{\ddagger}$ & 229 & 4.19 & 10.69 & $0-93$ & & NA & NA & NA & NA \\
\hline
\end{tabular}

NA: No aplica

Fuente: base de datos Línea Basal de Mortalidad Materna Guatemala 2000. Ministerio de Salud Pública y Asistencia Social de Guatemala 


\section{Cuadro II}

\section{Características sociodemográficas Relacionadas con 649 mUertes maternas hospitalarias Y No hospitalarias. Guatemala, 2000}

\begin{tabular}{|c|c|c|c|c|c|c|c|}
\hline \multirow[b]{2}{*}{ Variables } & \multicolumn{2}{|c|}{ Muertes totales $n=649$} & \multicolumn{2}{|c|}{ Muertes hospitalarias $n=270$} & \multicolumn{2}{|c|}{ Muertes no hospitalarias $n=379$} & \multirow[b]{2}{*}{ Valor $p$} \\
\hline & $n$ & $\%$ & $n$ & $\%$ & $N$ & $\%$ & \\
\hline \multicolumn{8}{|l|}{ Edad } \\
\hline$<$ de 19 años & 91 & 14.02 & 39 & 14.44 & 52 & 13.72 & 0.774 \\
\hline $20-24$ & 138 & 21.26 & 63 & 23.33 & 75 & 29.79 & \\
\hline $25-29$ & 114 & 17.57 & 47 & $17.4 \mid$ & 67 & 17.68 & \\
\hline $30-34$ & 116 & 17.87 & 48 & 17.78 & 68 & 17.94 & \\
\hline 35 o más & 190 & 29.28 & 73 & 27.04 & 117 & 30.87 & \\
\hline \multicolumn{8}{|l|}{ Grupo étnico } \\
\hline Indígena & 425 & 65.49 & 112 & 41.48 & 313 & 82.59 & 0.000 \\
\hline No indígena & 155 & 23.88 & 101 & 37.41 & 54 & 14.25 & \\
\hline Desconocido & 69 & 10.63 & 57 & 21.11 & 12 & 3.17 & \\
\hline \multicolumn{8}{|l|}{ Estado civil* } \\
\hline Casada/unida & 555 & 87.13 & 209 & 79.77 & 346 & 92.27 & 0.000 \\
\hline Nunca casada/soltera & 77 & 12.09 & 51 & 19.47 & 26 & 6.93 & \\
\hline Separada/viuda & 5 & 0.78 & 2 & 0.76 & 3 & 0.80 & \\
\hline \multicolumn{8}{|l|}{ Ocupación* } \\
\hline No remunerada & 599 & 94.78 & 229 & 88.76 & 370 & 98.93 & 0.000 \\
\hline Remunerada & 33 & 5.22 & 29 & 11.24 & 4 & $\mathrm{I} .07$ & \\
\hline \multicolumn{8}{|l|}{ Educación } \\
\hline Sin educación & 432 & 66.56 & 171 & 63.33 & 261 & 68.87 & 0.000 \\
\hline Primaria & 184 & 23.85 & 73 & 27.04 & III & 29.29 & \\
\hline Secundaria & 15 & 2.31 & II & 4.07 & 4 & 1.06 & \\
\hline Bachillerato & 9 & 1.39 & 9 & 3.33 & 0 & 0.00 & \\
\hline Universitaria & 9 & 1.39 & 6 & 2.22 & 3 & 0.79 & \\
\hline
\end{tabular}

Lugar de residencia (por regiones)

\begin{tabular}{|c|c|c|c|c|c|c|c|}
\hline Suroeste & 152 & 23.24 & 69 & 25.56 & 83 & 21.90 & 0.000 \\
\hline Noroeste & 148 & 22.80 & 33 & 12.22 & 115 & 30.34 & \\
\hline Norte & 101 & 15.56 & 20 & 7.41 & 81 & 21.37 & \\
\hline Metropolitana & 79 & 12.17 & 67 & 24.81 & 12 & 3.17 & \\
\hline Noreste & 56 & 8.63 & 22 & 8.15 & 34 & 8.97 & \\
\hline Central & 54 & 8.32 & 36 & 13.33 & 18 & 4.75 & \\
\hline Sureste & 33 & 5.08 & 18 & 6.67 & 15 & 3.96 & \\
\hline Petén & 26 & 4.01 & 5 & 1.85 & 21 & 5.54 & \\
\hline
\end{tabular}

Lugar donde ocurrió la muerte (por regiones)

\begin{tabular}{|c|c|c|c|c|c|c|c|}
\hline Noroeste & 144 & 22.19 & 29 & 10.74 & 115 & 30.34 & 0.000 \\
\hline Suroeste & 139 & 21.42 & 56 & 20.74 & 83 & 21.90 & \\
\hline Metropolitana & 135 & 20.80 & 123 & 45.56 & 12 & 3.17 & \\
\hline Norte & 95 & 14.64 & 14 & 5.19 & 81 & 21.37 & \\
\hline Noreste & 48 & 7.40 & 14 & 5.19 & 34 & 8.97 & \\
\hline Central & 40 & 6.16 & 22 & 8.15 & 18 & 4.75 & \\
\hline Petén & 26 & 4.01 & 5 & 1.85 & 21 & 5.54 & \\
\hline Sureste & 22 & 3.39 & 7 & 2.59 & 15 & 3.96 & \\
\hline
\end{tabular}

* Los totales de estas variables varían de acuerdo con los valores perdidos

Fuente: base de datos Línea Basal de Mortalidad Materna Guatemala 2000. Ministerio de Salud Pública y Asistencia Social de Guatemala 
Para ubicar el lugar donde se registró el caso de $\mathrm{MM}$, se agruparon los departamentos en ocho regiones, de acuerdo con la clasificación que utiliza el Ministerio de Salud Pública (suroeste, sureste, noroeste, noroeste, norte, Petén, central y metropolitana). La mayor parte de casos de MM sucedió en la zona noroeste. Se encontró que para los casos de MM hospitalaria la mayor proporción ocurrió en la zona metropolitana $(45.6 \%)$ y zona suroeste $(20.7 \%)$, mientras que casi todos los casos de MM no hospitalarias acontecieron en la zona noroeste $(30.3 \%)$, sureste $(21.9 \%)$ y norte $(21.4 \%)$ (cuadro II).

\section{Características obstétricas}

La media de embarazos en toda su vida fue mayor para el grupo de MM hospitalaria que para el grupo de MM no hospitalaria (3.5 y 4.6 embarazos, respectivamente). Esta diferencia tuvo significación estadística $(p<$ 0.05) (cuadro II).

$\mathrm{Al}$ analizar el periodo del embarazo (edad gestacional) al momento de la muerte materna, se encontró que la media de edad gestacional en MM hospitalarias (33.8 semanas) fue menor que la de las MM no hospitalarias (37.7 semanas); no obstante, en ambos grupos se presentó una importante proporción de datos desconocidos sobre el periodo de gestación (31.48\% y $20.54 \%$, respectivamente). En relación con las MM ocurridas antes de las 20 semanas de gestación, se encontró un total de $6.3 \%$, con predominio de casos de MM hospitalaria comparados con las MM no hospitalarias (11.1\% y $2.9 \%$, respectivamente). Esta diferencia fue estadísticamente significativa $(p<0.05)$ (cuadros I y III).

Referente a la persona que atendió el parto de los casos de MM, el médico fue sobre todo la persona que asistió los casos de MM hospitalaria (68.52\%), contrario a lo que ocurrió en los casos de MM no hospitalaria donde la mayor proporción de partos estuvo en manos de una partera tradicional (comadrona) $(59.89 \%)$, seguida por familiares (28.23\%) (cuadro III).

El principal resultado del embarazo, tanto en el grupo de MM hospitalaria como en el de MM no hospitalaria, fue nacido vivo $(58.2 \%$ y $75.2 \%$, respectivamente). Al analizar la causa básica de muerte en los casos de MM hospitalaria se encuentra que la mayoría de mujeres murió por hemorragia $(36.7 \%)$, seguida de las infecciones (26.5\%); en el grupo no hospitalario la mayores causas de muerte fueron hemorragia $(74.6 \%)$ e infecciones $(12.5 \%)$. Estas diferencias tuvieron significación estadística $(p<0.05)$ (cuadro III).

La media del tiempo transcurrido entre la resolución del embarazo y la muerte materna fue estadísticamente diferente entre ambos grupos -hospitalario
5.59 días y no hospitalario 1.93 días- $(p<0.05)$. La mayor proporción de muertes se presentó el mismo día del parto, tanto para las muertes hospitalarias como para las no hospitalarias $(33.3 \%$ y $66.5 \%$, respectivamente). Para el grupo de muertes hospitalarias se analizó el periodo de tiempo que transcurrió entre el ingreso hospitalario y la muerte, y se encontró que en promedio fue de 4.19 días (cuadros I y III).

En el certificado de defunción la causa de muerte se encontró asociada al embarazo en $56.7 \%$ de los casos de muerte hospitalaria y en $55.7 \%$ de los casos de muerte no hospitalaria. Fueron médicos quienes emitieron los certificados en casi todos los casos de las muertes hospitalarias $(96.25 \%)$, comparado con casi la mitad de los casos de muertes no hospitalarias (53.7\%).

Se realizó autopsia verbal en $46.30 \%$ de los casos del grupo hospitalario y en $82.59 \%$ del grupo no hospitalario. Según la clasificación internacional de las enfermedades (CIE, 10 a edición), la defunción obstétrica directa fue en ambos grupos la más frecuente (84.07 y 93.93\%, respectivamente) (cuadro IV).

\section{Factores relacionados con la mortalidad materna no hospitalaria}

Si se comparan las muertes maternas ocurridas en el hospital (MM hospitalaria) con las muertes maternas ocurridas fuera del hospital (MM no hospitalaria, hogar, calle, otro), se reconoce que los factores relacionados con la probabilidad de morir fuera del hospital, ajustados en el modelo multivariado, fueron: edad menor de 20 años de edad, en comparación con las mujeres de 25 a 29 años ( $R M=2.0$; IC95\% 0.8-5.3); pertenecer a una etnia indígena implica tres veces más posibilidad de morir fuera del hospital que no serlo ( $R M=3.4$; IC95\% 2.0-5.3) (cuadro V).

Carecer de una ocupación remunerada incrementa nueve veces la posibilidad de morir fuera del hopital $(R M=8.9$; IC95\% 1.7-46.4). La posibilidad de MM no hospitalaria es el doble si la mujer estuvo privada de educación o tiene la primaria incompleta $(R M=2.0$; IC95\% 1.0-3.8) (cuadro V).

El antecedente de cuatro o más embarazos previos implicó un riesgo 1.6 veces mayor de MM no hospitalaria, aunque resultó marginalmente significativo comparado con el de las mujeres que presentaban el antecedente de uno a tres embarazos ( $R M=1.6 ;$ IC95\% 0.8-3.3). Quienes obtuvieron un producto a término ( 38 o más semanas de gestación) exhibieron un riesgo tres veces mayor de MM no hospitalaria $(R M=3.6$; IC $95 \%$ 1.4-9.3), comparado con el de las que llegan a menos de 20 semanas de gestación. La hemorragia incrementa cuatro veces la posibilidad de MM no hospitalaria 


\section{Cuadro III \\ Características obstétricas relacionadas con 649 muertes maternas hospitalarias Y No hospitalarias. Guatemala, 2000}

\begin{tabular}{|c|c|c|c|c|c|c|c|}
\hline \multirow[b]{2}{*}{ Variables } & \multicolumn{2}{|c|}{$\begin{array}{c}\text { Muertes totales } \\
n=649\end{array}$} & \multicolumn{2}{|c|}{$\begin{array}{c}\text { Muertes hospitalarias } \\
n=270\end{array}$} & \multicolumn{2}{|c|}{$\begin{array}{c}\text { Muertes no hospitalarias } \\
n=379\end{array}$} & \multirow[b]{2}{*}{ Valor $p$} \\
\hline & $n$ & $\%$ & $N$ & $\%$ & $n$ & $\%$ & \\
\hline \multicolumn{8}{|c|}{ Número de gestaciones previas* } \\
\hline Ninguno & 94 & 15.36 & 47 & 19.50 & 47 & 12.67 & 0.003 \\
\hline I-3 embarazos & 207 & 33.82 & 91 & 37.76 & 116 & 31.27 & \\
\hline 4 o más embarazos & 311 & 50.82 & 103 & 42.74 & 208 & 56.06 & \\
\hline
\end{tabular}

Periodo del embarazo al momento de la muerte materna

\begin{tabular}{|c|c|c|c|c|c|c|c|}
\hline Menor a 20 sem. (aborto) & 41 & 6.32 & 30 & II.II & II & 2.90 & 0.000 \\
\hline De 20 a 27 sem. (parto producto inmaduro) & 19 & 2.93 & 6 & 2.22 & 13 & 3.43 & \\
\hline De 28 a 37 sem. (parto producto prematuro) & 66 & 10.17 & 40 & $|4.8|$ & 26 & 6.86 & \\
\hline De 38 a 4 I sem. (parto producto a término) & 345 & 53.16 & 105 & 38.89 & 240 & 63.32 & \\
\hline 42 o más semanas (parto postérmino) & 14 & 2.16 & 4 & 1.48 & 10 & 2.64 & \\
\hline Desconocido & 164 & 25.27 & 85 & 31.48 & 79 & 20.84 & \\
\hline
\end{tabular}

Resultado del embarazo

\begin{tabular}{|c|c|c|c|c|c|c|}
\hline Nació vivo & 442 & 68.10 & 157 & 58.15 & 285 & 75.20 \\
\hline Nació muerto & 63 & 9.71 & 37 & 13.70 & 26 & 6.86 \\
\hline Aborto & 61 & 9.40 & 39 & 14.44 & 22 & 5.80 \\
\hline No nació & 38 & 5.86 & 18 & 6.67 & 20 & 5.28 \\
\hline Embarazo ectópico & 4 & 0.62 & 4 & 1.48 & 0 & 0.00 \\
\hline Desconocido & 41 & 6.32 & 15 & 5.56 & 26 & 6.86 \\
\hline
\end{tabular}

Lugar donde finalizó el embarazo

\begin{tabular}{|c|c|c|c|c|c|c|c|}
\hline No hospital & 385 & 59.32 & 46 & 17.04 & 339 & 89.45 & 0.000 \\
\hline Hospital & 200 & 30.82 & 186 & 68.89 & 14 & 3.69 & \\
\hline Desconocido & 64 & 9.86 & 38 & 14.07 & 26 & 6.86 & \\
\hline
\end{tabular}

\begin{tabular}{lrrrrrr}
$\begin{array}{l}\text { Personal que atendió el parto } \\
\text { Comadrona }\end{array}$ & 264 & 40.68 & 37 & 13.70 & 227 & 59.89 \\
\hline Médico & 202 & 31.12 & 185 & 68.52 & 17 \\
\hline Familiar & 132 & 20.34 & 25 & 9.26 & 107 & 28.23 \\
\hline Desconocido & 51 & 7.86 & 23 & 8.52 & 28
\end{tabular}

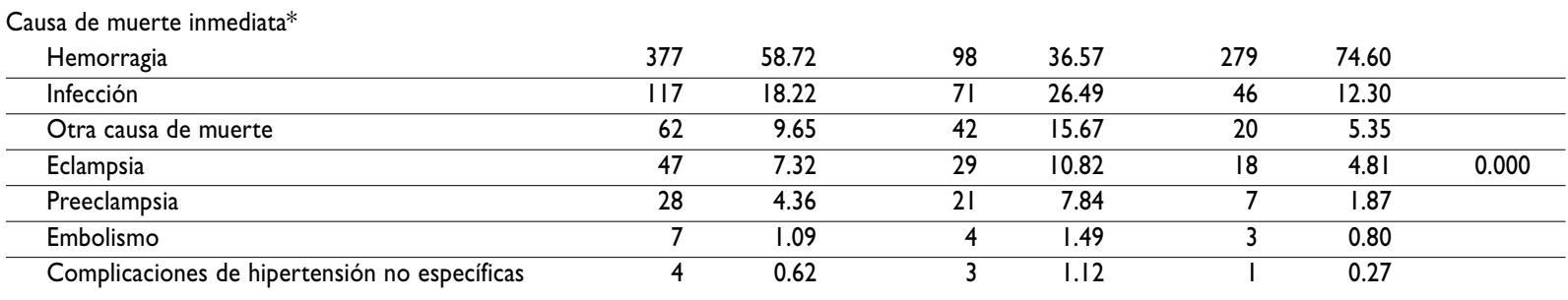

Tiempo entre resolución del embarazo y muerte (días)

\begin{tabular}{|c|c|c|c|c|c|c|c|}
\hline El mismo día & 342 & 52.70 & 90 & 33.33 & 252 & 66.49 & 0.000 \\
\hline Menos de 48 horas & 76 & II.7I & 44 & 16.30 & 32 & 8.44 & \\
\hline Menos de 7 días & 58 & 8.49 & 44 & 16.30 & 14 & 3.69 & \\
\hline Más de 7 días & 65 & 10.02 & 35 & 12.96 & 30 & 7.92 & \\
\hline Desconocidos & 108 & 16.64 & 57 & 21.11 & 51 & 13.46 & \\
\hline
\end{tabular}

* Los totales de estas variables varían de acuerdo con los valores perdidos

Fuente: base de datos Línea Basal de Mortalidad Materna Guatemala 2000. Ministerio de Salud Pública y Asistencia Social de Guatemala 


\section{Cuadro IV \\ Datos del CERTIFICADO de defUnCIÓN RELACIONADOS CON 649 MUERTES MATERNAS hospitalaRIAS Y NO hospitalarias. Guatemala, 2000}

\begin{tabular}{|c|c|c|c|c|c|c|c|}
\hline \multirow[b]{2}{*}{ Variables* } & \multicolumn{2}{|c|}{ Muertes totales $n=649$} & \multicolumn{2}{|c|}{ Muertes hospitalarias $n=270$} & \multicolumn{2}{|c|}{ Muertes no hospitalarias $n=379$} & \multirow[b]{2}{*}{ Valor } \\
\hline & $n$ & $\%$ & $N$ & $\%$ & $n$ & $\%$ & \\
\hline \multicolumn{8}{|c|}{ Persona que certifica la defunción } \\
\hline Médico & 438 & 72.52 & 257 & 96.25 & 181 & 53.71 & 0.000 \\
\hline Empírico & 89 & 14.74 & 8 & 3.00 & 69 & 20.47 & \\
\hline Autoridad & 77 & 12.75 & 2 & 0.75 & 87 & 25.82 & \\
\hline
\end{tabular}

Causa de muerte en certificado de defunción

\begin{tabular}{lrrrrrrr} 
Asociada al embarazo & 364 & 56.09 & 153 & 56.67 & 211 & 55.67 & 0.494 \\
\hline No se menciona el embarazo & 232 & 35.75 & 99 & 36.67 & 133 & 35.09 & \\
\hline No existe certificado & 53 & 8.17 & 18 & 6.67 & 35 & 9.23
\end{tabular}

Autopsia verbal

\begin{tabular}{llllllll} 
Sí & 438 & 67.49 & 125 & 46.30 & 313 & 82.5 & 0.000 \\
\hline No & 211 & 32.51 & 145 & 53.70 & 66 & 17.41 &
\end{tabular}

\begin{tabular}{lrrrrrrr} 
Clasificación final de la muerte & & & & & & \\
Defunción obstétrica directa & 583 & 89.83 & 227 & 84.07 & 356 & 93.93 & 0.000 \\
\hline Defunción obstétrica indirecta & 66 & 10.17 & 43 & 15.93 & 23 & 6.07
\end{tabular}

* Los totales de estas variables varían de acuerdo con los valores perdidos

Fuente: base de datos Línea Basal de Mortalidad Materna Guatemala 2000. Ministerio de Salud Pública y Asistencia Social de Guatemala

comparada con otras causas básicas de muerte $(R M=$ 4.3; IC95\% 2.3-7.9).

La zonas geográficas de procedencia de los casos de MM representaron un mayor riesgo de MM hospitalaria en la región norte y el Petén, comparadas con el área metropolitana de la ciudad de Guatemala $(R M=$ 4.2 ; IC95\% 1.8-10.0) (cuadro V).

\section{Discusión}

Los resultados obtenidos del análisis realizado en este estudio muestran que las características de las mujeres que fallecieron en la República de Guatemala por causa materna en el año 2000 se comportaron de diferente manera en los grupos estudiados -hospitalario y no hospitalario-.

La mayor proporción de muertes maternas fueron no hospitalarias; esto puede estar relacionado con el hecho de que una alta proporción de esta población habita en áreas rurales o de alta marginalidad, en las que se tienen limitantes para el acceso a centros de salud, donde la atención del parto está en manos de perso- nal capacitado, y donde se dan condiciones culturales que pueden influir para que el mayor porcentaje de partos, y por tanto de muertes, ocurra en lugares distintos a los hospitales. Similares hallazgos comunicó Kestler en Guatemala en 1986, ${ }^{10}$ que difieren de lo publicado en países como República Dominicana, donde la mayor proporción de partos ocurre en los hospitales, y por tanto el número de muertes maternas es mayor en estos sitios. ${ }^{4,11,12}$

Se encontró que la mayor proporción de $\mathrm{MM}$ ocurre en mayores de 35 años de edad, tanto a nivel hospitalario como no hospitalario, y ésta es una realidad que se ha mantenido en los últimos años, y que es consistente con otros informes como el estudio de Kestler y Ramírez, en Guatemala, del periodo $1993-1996,{ }^{13}$ en que identificaron esta edad como la de mayor riesgo. La literatura internacional respalda el hecho de que esta complicación se presenta con mayor agudeza en los extremos de la edad reproductiva (menores de 20 años y mayores de 35 años). ${ }^{2,4,11,14,15}$

Entre los hallazgos destaca el hecho que sean las mujeres indígenas -tanto en muertes hospitalarias 


\section{Cuadro V}

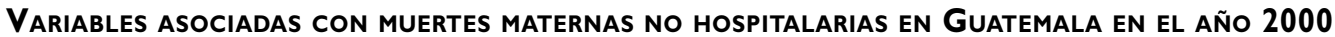

\begin{tabular}{|c|c|c|c|c|c|}
\hline \multirow[b]{2}{*}{ Variables } & \multirow{2}{*}{$\begin{array}{c}\text { Total } \\
n=649\end{array}$} & \multicolumn{2}{|c|}{ RM ajustada por edad } & \multicolumn{2}{|c|}{ RM ajustada* } \\
\hline & & $R M$ & IC95\% & $\overline{R M}$ & $1 C 95 \%$ \\
\hline \multicolumn{6}{|l|}{ Edad (años) } \\
\hline$<19$ años & 91 & 0.93 & $0.53-1.63$ & 2.06 & $0.80-5.30$ \\
\hline 20-24 años & 138 & 0.83 & $0.51-1.38$ & 1.07 & $0.47-2.41$ \\
\hline $25-29$ años & 114 & I & & I & \\
\hline 30-34 años & 116 & 0.99 & $0.59-1.68$ & 0.95 & $0.40-2.23$ \\
\hline$>35$ años & 190 & 1.12 & $0.70-1.81$ & 0.57 & $0.25-1.27$ \\
\hline
\end{tabular}

Grupo étnico

\begin{tabular}{|c|c|c|c|c|}
\hline No indígena & 155 & I & I & \\
\hline Indígena & 425 & $5.19 \quad 3.49-1.03$ & 3.42 & $2.0-5.30$ \\
\hline
\end{tabular}

Estado civil ${ }^{\ddagger}$

\begin{tabular}{|c|c|c|c|c|c|}
\hline Con pareja & 555 & I & & I & \\
\hline Sin pareja & 82 & 0.34 & $0.21-0.55$ & 0.75 & $0.33-1.73$ \\
\hline \multicolumn{6}{|l|}{ Ocupación $\ddagger$} \\
\hline Remunerada & 33 & I & & I & \\
\hline No remunerada & 599 & 11.48 & $3.98-33.12$ & 8.95 & $1.73-46.36$ \\
\hline
\end{tabular}

Educación

\begin{tabular}{lrrrrr} 
Mayor a primaria & 97 & 1 & 1 & \\
\hline Sin educación/primaria incompleta & 552 & 2.73 & $1.73-4.28$ & 1.96 & $1.00-3.83$
\end{tabular}

Lugar de residencia (regiones)

\begin{tabular}{lccccc} 
Sureste & 33 & 2.87 & $1.29-6.37$ & 3.88 & $1.08-13.91$ \\
\hline Noreste & 56 & 5.24 & $2.68-10.28$ & 5.55 & $0.23-6.33$ \\
\hline Norte y Petén & 127 & 13.93 & $7.66-25.32$ & 4.25 & $1.80-10.00$ \\
\hline Central y Metropolitana & 133 & 1 & & & 1 \\
\hline Noroeste & 148 & 11.86 & $6.76-20.82$ & 3.12 & $1.29-7.57$ \\
\hline Suroeste & 152 & 4.12 & $2.45-6.91$ & 2.37 & $1.06-5.31$
\end{tabular}

Número de gestaciones previas $\ddagger$

\begin{tabular}{lccccc} 
Ninguna & 94 & $0.7 \mathrm{I}$ & $0.43-1.18$ & 0.88 & $0.38-2.0 \mathrm{I}$ \\
\hline I-3 embarazos & 207 & $\mathrm{I}$ & & $\mathrm{I}$ & \\
\hline 4 o más embarazos & $3 \mathrm{II}$ & $2.1 \mathrm{I}$ & $\mathrm{I} .32-3.38$ & $\mathrm{I} .59$ & $0.77-3.28$
\end{tabular}

Periodo del embarazo al momento de la muerte ${ }^{\ddagger}$

Menos de 20 semanas (aborto)

\begin{tabular}{rllll}
41 & $\mathrm{I}$ & $\mathrm{I}$ \\
85 & 2.30 & $1.02-5.18$ & 3.45 & $1.20-9.92$ \\
\hline 360 & 6.19 & $2.99-12.82$ & 3.65 & $1.43-9.28$
\end{tabular}

Causa de muerte inmediata ${ }^{\ddagger}$

\begin{tabular}{llllll} 
Hemorragias & 377 & 5.82 & $3.83-8.83$ & 4.28 & $2.32-7.91$ \\
\hline Infecciones & 117 & 1.31 & $0.79-2.17$ & 1.20 & $0.58-2.49$ \\
\hline Otros problemas & 148 & $\mathrm{I}$ & & $\mathrm{I}$ &
\end{tabular}

\footnotetext{
* Datos ajustados por todas las variables incluidas en el modelo

‡ Los totales de estas variables varían de acuerdo con los valores perdidos
}

Fuente: base de datos Línea Basal de Mortalidad Materna Guatemala 2000. Ministerio de Salud Pública y Asistencia Social de Guatemala 
como no hospitalarias- el grupo más vulnerable. En Guatemala la población indígena constituye $48 \%$ de la población total, y habita en áreas rurales o marginales de las ciudades importantes; de manera predominante presenta niveles de pobreza extrema, grado de educación bajo, factores éstos que, al conjugarse con otros aspectos culturales de las poblaciones indígenas que las pone en desventaja, son limitantes para el acceso a los servicios de salud, todo lo cual los convierte en un grupo de alto riesgo. A pesar de que se han implementado acciones en busca de mejorar la salud de estas mujeres, este grupo sigue comportándose como el de mayor riesgo. Reducir la mortalidad materna en este grupo es una meta con bastante grado de dificultad en países como Guatemala, donde los aspectos culturales suelen ser importantes barreras por superar. ${ }^{10,16,17}$

La causa principal de muerte fue hemorragia, tanto en el grupo hospitalario como en el no hospitalario. La infección fue la segunda causa de muerte en el grupo hospitalario. Estos resultados concuerdan con lo que refiere la literatura en relación con las muertes maternas, en el sentido de que disminuirían con sólo garantizar una atención obstétrica de calidad, sobre todo al momento del parto, y que éste se dé en condiciones adecuadas, con el insumo médico mínimo y con la atención de personal de salud calificado. ${ }^{2,5,6,18}$

En muertes no hospitalarias la mayor proporción de mujeres residía y murió en la región noroeste; este dato es importante dado que esta región está constituida únicamente por dos departamentos -Huehuetenango y Quiché-, considerados con alto grado de marginación. En estos casos fueron comadronas y familiares quienes asistieron el parto. Se considera que la inaccesibilidad a los servicios de la salud, la baja calidad de atención recibida en el parto, los niveles altos de pobreza y las barreras culturales son condiciones que pueden influir en este comportamiento. ${ }^{2,5,6,18}$

Es importante destacar que en todos los casos de MM la mayor proporción de las muertes se presentó el mismo día en el que finalizó el embarazo -MM no hospitalaria 33\% y MM hospitalaria $66 \%-$, lo que concuerda con lo que informan las publicaciones acerca de que la atención de emergencias obstétricas, la atención por personal capacitado y el control posparto son factores importantes a considerar para reducir la mortalidad materna. $5,13,19,20$

Los factores relacionados con las muertes maternas no hospitalarias son similares a los descritos en un estudio realizado por Kestler, en Guatemala, en 1986, y con estudios efectuados en otras regiones ${ }^{2,4,10}$-mujeres mayores de 35 años, indígenas, con ocupación no remunerada, sin educación o con primaria incompleta, con embarazo a término, cuyo producto haya nacido vivo, atendido por comadrona, y con diagnóstico final de hemorragia son las que mayor riesgo corren de morir-, aunque al ajustar estas variables se encuentra que algunos de estos factores se modifican por el efecto de los demás, y así se reconocen como datos relevantes que el riesgo se incrementa dos veces en mujeres de 30 a 34 años, indígenas, sin pareja, pero que el riesgo de morir se incrementa ocho veces si se tiene una ocupación no remunerada.

Por otra parte, también se ha estudiado el efecto de la accesibilidad a los servicios y la utilización oportuna de los mismos. Así, se han identificado la distancia y los tiempos de transportación y de espera como obstáculos para obtener la atención requerida y, en consecuencia, como factores de riesgo para la muerte materna. De esta manera, las mujeres con mayor nivel socioeconómico cuentan con más posibilidades para que se les detecten problemas en el embarazo y recibir atención a tiempo, con mejores perspectivas de sobrevida.

Las muertes que no se verifican en el ámbito hospitalario podrían reflejar características más adversas en el contexto social y geográfico, que necesitan estudiarse.

Como limitaciones del estudio se puede considerar el hecho de que los datos empleados en el mismo se obtuvieron de registros civiles, certificados de defunción y autopsias verbales de muertes de mujeres en edad reproductiva en Guatemala durante el año 2000, por lo que se considera que esta información pueda tener algunas diferencias entre el comportamiento de la mortalidad materna hospitalaria y no hospitalaria.

Aunado a lo anterior, el retraso y subregistro en la información pueden también generar limitantes en el análisis comparativo entre muertes hospitalarias y no hospitalarias (sesgo de información), como queda de manifiesto en la proporción de respuesta desconocida.

El hecho que los datos se obtuvieran por medio de autopsia verbal también pudo ocasionar sesgos de memoria al obtener la información..$^{21-22}$

Estas limitantes de errores y deficiencias en la obtención de información se compensaron con el análisis inferencial estadístico multivariado, que incluyó ajuste por todas las variables potencialmente confusoras.

\section{Agradecimiento}

A las autoridades del Ministerio de Salud Pública y Asistencia Social de Guatemala su colaboración en la realización del presente estudio, al permitir el uso de la base de datos de la Línea Basal de Mortalidad Ma- 
terna (LBMM) 2000, en especial al doctor Roberto Santizo, director del Programa de Salud Reproductiva, durante el periodo en que se realizó este estudio.

\section{Referencias}

I. Organización Mundial de la Salud. Maternidad Saludable. Disponible en: http://www.col.ops-oms.org/familia/maternidad.htm. [Consultado 2003 feb 8].

2. Lozano R, Hernández B, Langer A. Factores sociales y económicos de la mortalidad materna en México. Foro Mundial de Salud Reproductiva. Curso electrónico: cultura, salud y reproducción. Disponible en: http:// www.hsph.harvard.edu/Organizations/healthnet/_Spanish/course/ sesion6/lozano.html. [Consultado 2003 feb 6].

3. Organización Panamericana de la Salud. Plan Estratégico de Mortalidad Materna. Disponible en: http://»http://www.ops.org.ni/ opsnic/tematicas/s-sexual/downloads/plan-estrat-mortalidadmaterna.pdf» www.ops.org.ni/opsnic/tematicas/s-sexual/downloads/ plan-estrat-mortalidad-materna.pdf. [Consultado 2003 agosto 24]. 4. Schwarcz R. Maternal mortality in Latin America and the Caribbean. Lancet 2000;356 suppl SI I:3245-3267.

5. Organización Panamericana de la Salud. Reducir la mortalidad materna es prioridad diaria de la OPS. Información de Prensa. Mayo 2002. Disponible en: http://www.paho.org/Spanish/DPI/I00/ I00feature 19.htm. [Consultado 2003 mayo 9]. 6. World Health Organization. Reduction of maternal mortality. Anoint WHO/UNFPA/UNICEF/World Bank Statement. Ginebra:WHO, 1999.

7. Aguilar N. Hechos y reflexiones sobre la mortalidad materna. Revista Asociación Antioqueña de Obstetricia y Ginecología 2001;17-18.

8. Instituto Nacional de Estadística. Base de Datos de Nacimientos 2000. Guatemala: INE, 2002.

9. Organización Panamericana de la Salud. Clasificación Internacional de Enfermedades y Problemas Relacionados con la Salud. 10 $0^{\mathrm{a}}$. Revision. Washington, D.C.: OPS 1995. Pub. Científica No. 554.
10. Kestler E. Guatemala: maternal mortality in Guatemala: assessing the gap, beginning to bridge it. World Health Stat Q 1995;48:28-33.

II. Hernández B, Langer A, Romero M, Chirinos J. Factores asociados a la muerte materna hospitalaria en el estado de Morelos, México. Salud Publica Mex 1994;36(5):52I-528.

12. Secretaria de Salud Pública y Asistencia Social, USAID, Population Council. Strategic Assessment of Reproductive Health in the Dominican Republic. [Final Report]. Population Council. Santo Domingo;2002. 13. Kestler E, Ramírez L. Pregnancy related mortality in Guatemala 1993-996. Rev Panam Salud Publica 2000; 7(I):41-46.

14. Reyes S. Mortalidad materna en México. México: Subdirección General Médica. IMSS. Jefatura de Servicios de Investigación Médica, 1994.

15. Salazar A, Vásquez M. Mortalidad materna en Cali iuna década sin cambios? Colomb Med 1996;27(3-4): I 17-I 24.

16. Organización Mundial de la Salud. Mortalidad materna: ayudar a las mujeres a evitar el camino de la muerte. WHO Chron 1986;40(5): 195-205.

17. Fuentes A, Pages D, et. al. Mortalidad materna: algunos factores a considerar. 1986-1995. Revista Cubana de Obstetricia y Ginecología 1998;24 (2):80-85.

18. Rendon L, Langer A, Hernández B. Women's living conditions and maternal mortality in Latin America. Bull of Saint Panam 1993;27(I):56-64.

19. Network. Elementos claves para reducir la mortalidad materna. Boletín trimestral de salud. 2002. 22(2). Disponible en: http:// www.reproline.jhu.edu.-/spanish/6read/6issues/network/v22-2/ ns 2221 I.html.

20. Network. Una mejor atención en el posparto salva vidas. Boletín trimestral de salud. 1997.17(4). Disponible en: http:// www.reproline.jhu.edu.-/spanish/6read/6issues/network/v17-4/ nt I744s.html.

21. Sloan N, Langer A, Hernández B, Romero M, Winikoff B. The etiology of maternal mortality in developing countries: what do verbal autopsies tell us. Bull World Health Organ 200I; 79(9):805-810.

22. Langer $A$. Identifying interventions to prevent maternal mortality in Mexico. A multi-center verbal autopsies study. México, DF: National Safe Motherhood Committee of Mexico, 1998. [Ponencia]. 